\title{
Insights into Dissolution and Solution Chemistry of Multidrug Formulations of Antihypertensive Drugs
}

Mira El Sayed ${ }^{1,2}$, Amjad Alhalaweh², and Christel A.S. Bergström ${ }^{1 *}$

${ }^{1}$ Department of Pharmacy, Uppsala University, Biomedical Centre, SE-751 23 Uppsala, Sweden.

${ }^{2}$ Recipharm OT Chemistry AB, SE-754 50 Uppsala, Sweden.

*Address correspondence to:

Prof. Christel Bergström

Department of Pharmacy

Uppsala University

P.O. Box 580

SE-751 23 Uppsala, Sweden

Email: christel.bergstrom@,farmaci.uu.se

Phone: $+46-184714118$

Fax: $+46-184714223$ 


\section{Table of contents}

Table S1. Mobile phase composition of the gradient elution for HPLC analysis of CLD.

Figure S1. Mean intensity (kcps) determined by DLS as a function of the drug concentration for a) FDN b) NIF

Figure S2. Characterization of the crystalline form of FDN by DSC and PXRD. (a) DSC heating curve of FDN as received. (b) PXRD pattern of FDN as received.

Figure S3. Characterization of the crystalline form of NIF by DSC and PXRD. (a) DSC heating curve of NIF as received. (b) PXRD pattern of NIF as received.

Figure S4. Characterization of the crystalline form of CLD by DSC and PXRD. (a) DSC heating curve of CLD as received. (b) PXRD pattern of CLD as received.

Figure S5. Characterization of the crystalline form of IPM by DSC and PXRD. (a) DSC heating curve of IPM as received. (b) PXRD pattern of IPM as received.

Figure S6. DSC heating curve of MTN as received.

Figure S7. Characterization of the formulation containing 80\% HPMCAS and 20\% drugs (1:1 mole ratio of FDN and IPM) by PXRD.

Figure S8. Characterization of the formulation containing 80\% PVP and 20\% drugs (1:1 mole ratio of FDN and IPM) by PXRD.

Figure S9. DSC thermograms of the FDN and MTN physical mixture at different molar ratios.

Figure S10. Glass transition temperature $\left(\mathrm{T}_{\mathrm{g}}\right)$ of a miscible system of FDN and MTN as a function of MTN weight fraction. Dashed line represents the predicted $\mathrm{T}_{\mathrm{g}}$ based on Fox equation.

Figure S11. DSC heating curves of the HPMCAS-FDN-IPM 1:1 mole ratio (green curve), HPMCASFDN-IPM 2:1 mole ratio (blue curve) and PVP-FDN-IPM 1:1 mole ratio (maroon curve) at the end of the dissolution study.

Figure S12. Dissolution profiles of formulations containing 80\% HPMCAS and 20\% drugs (2:1 mole ratio of FDN and IPM) in buffer (a) FDN and (b) IPM. Error bars show standard deviation, $n=3$. Error bars for some points are smaller than the symbols.

Figure S13. Concentration of FDN (green $\triangle$ ) and RTV (orange $\triangle$ ) in the supernatant layer using the antisolvent method as a function of the mole fraction of RTV in the solution. Dashed lines represent the predicted concentrations based on Eq. 2 (three replicates).

Figure S14. Dissolution profiles of formulations containing 80\% PVP and 20\% drugs (1:1 molar ratio of FDN and IPM) in buffer (a) FDN in green and (b) IPM in blue. Error bars (three replicates) show standard deviations (error bars are smaller than the symbols for some measurements). 
Table S1. Mobile phase composition of the gradient elution for HPLC analysis of CLD.

\begin{tabular}{ccc}
\hline Time $(\mathrm{min})$ & Solvent $^{*}(\%)$ & Solvent B $^{*}(\%)$ \\
\hline 0 & 0 & 100 \\
0.2 & 10 & 90 \\
3 & 90 & 10 \\
7.1 & 10 & 90 \\
\hline
\end{tabular}

*A: water, B: acetonitrile 
(a)

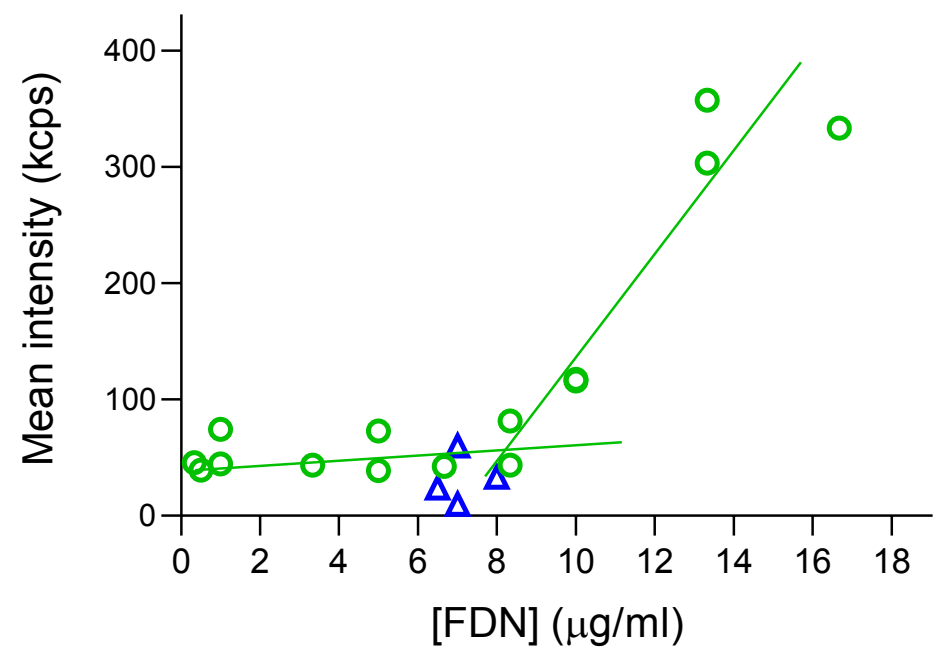

(b)

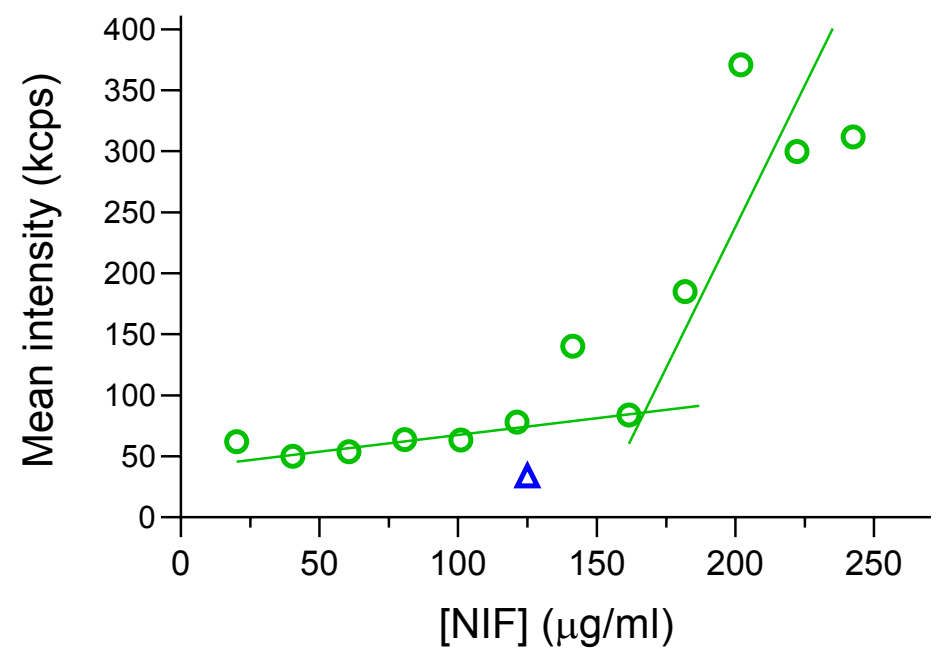

Figure S1. Mean intensity (kcps) as a function of the drug concentration for a) FDN: green circles represent solutions of FDN at different concentrations. Blue triangles represent the supernatant samples from the FDN-IPM antisolvent experiment after centrifugation by the Heraeus Biofuge b) NIF in phosphate buffer green circles represent solutions of NIF at different concentrations. Blue triangle represents the supernatant sample from the NIF AS determination experiment after centrifugation by the Heraeus Biofuge. The DLS analysis was performed at different drug concentrations using Litesizer 500 instrument and Kalliope ${ }^{\mathrm{TM}}$ particle analysis software (Anton Paar, Graz, Austria). Experiments were performed using disposable polystyrene cuvettes. 
(a)

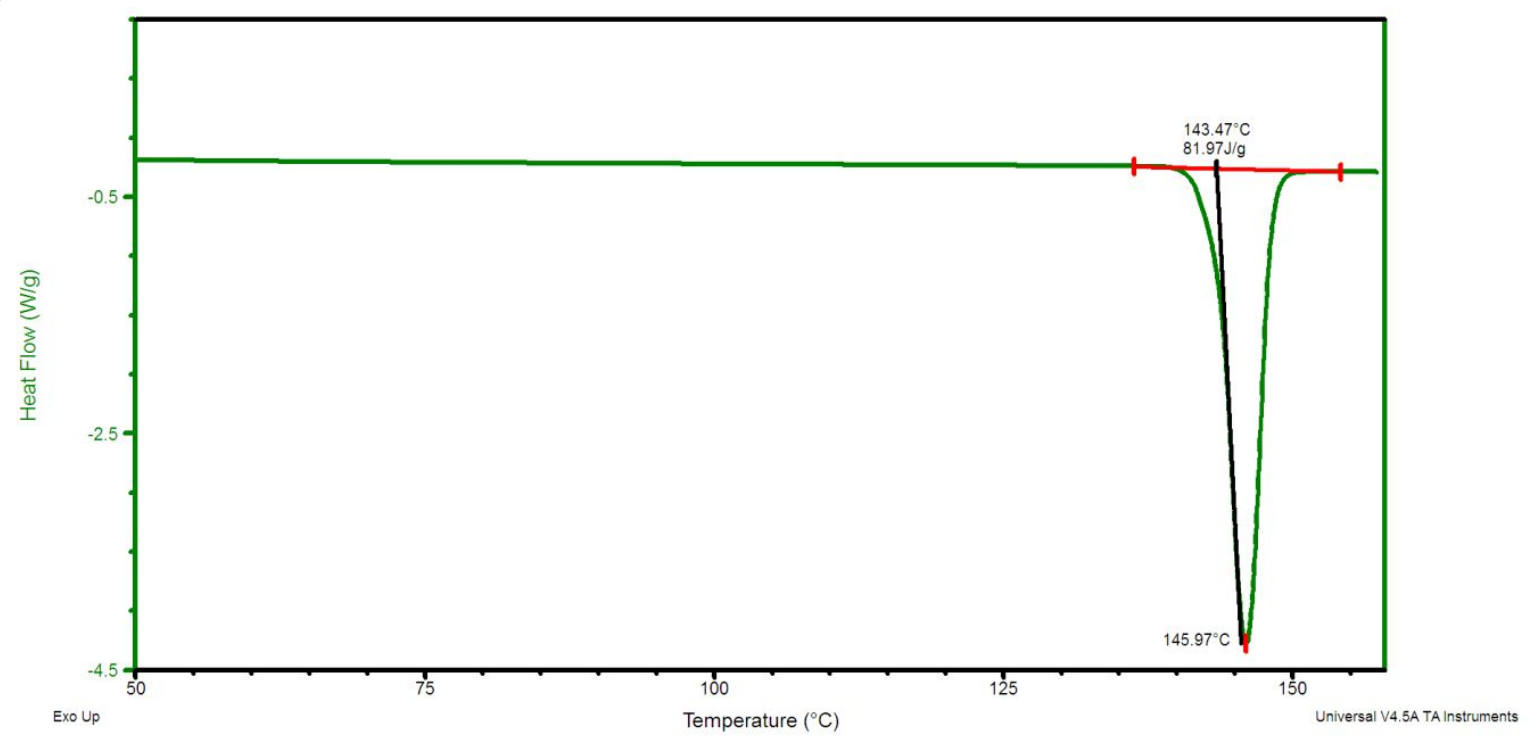

(b)

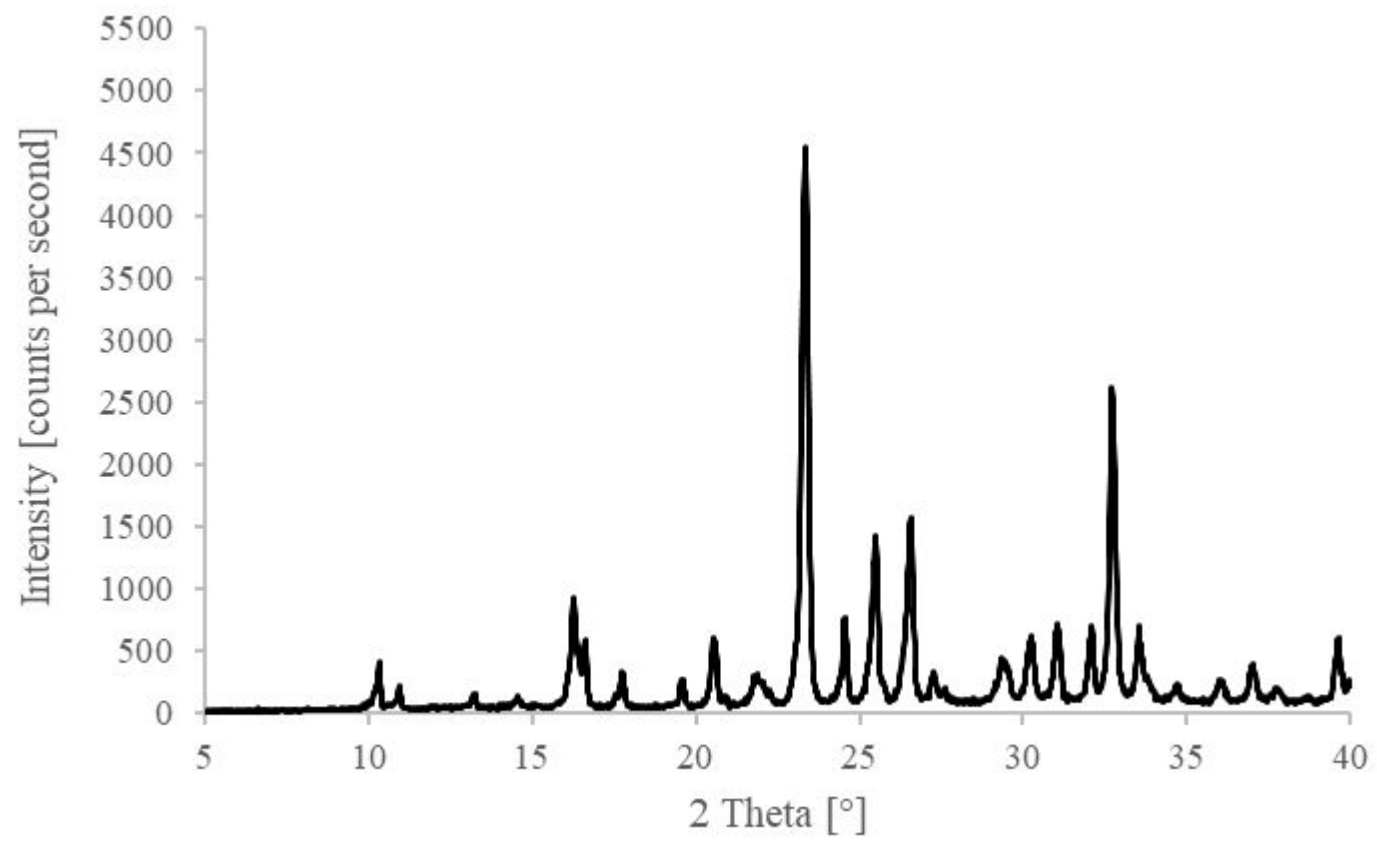

Figure S2. Characterization of the crystalline form of FDN by DSC and PXRD. (a) DSC heating curve of FDN as received. (b) PXRD pattern of FDN as received. 
(a)

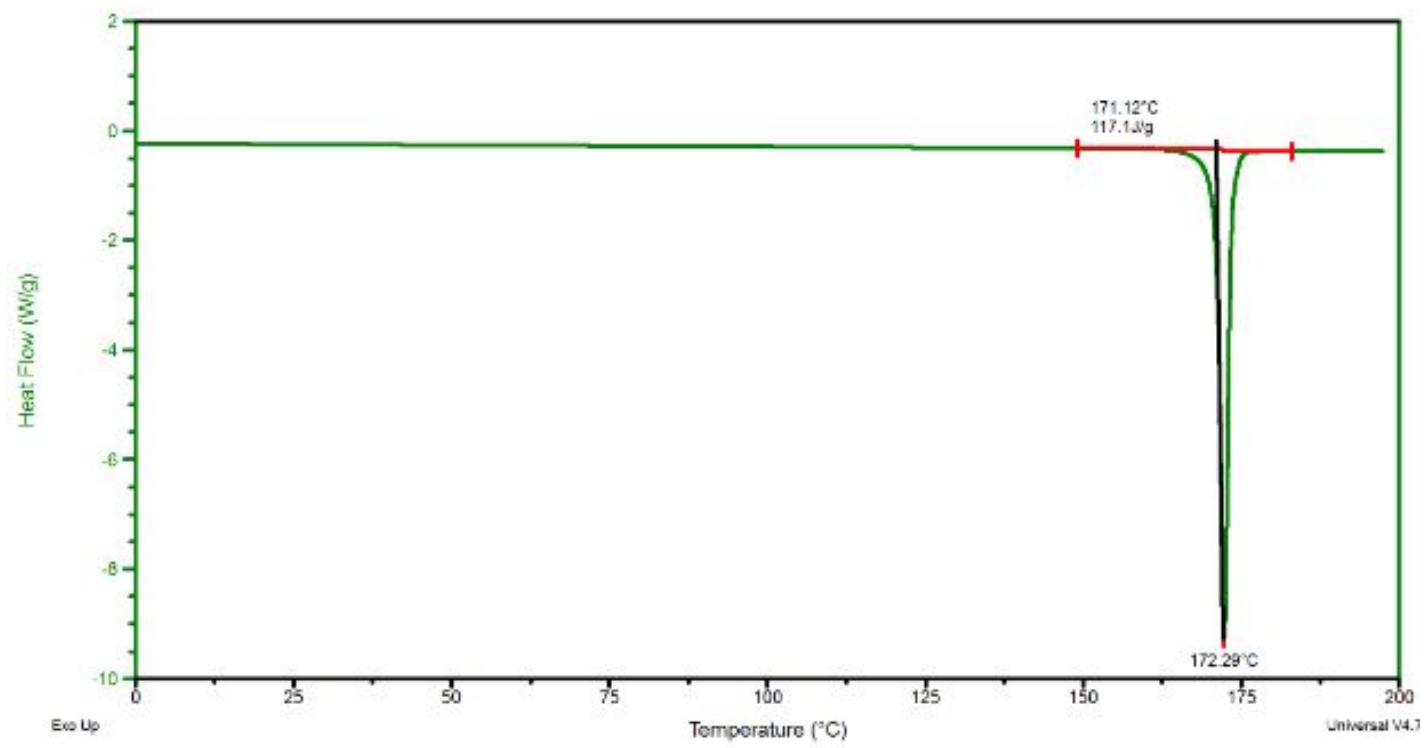

(b)

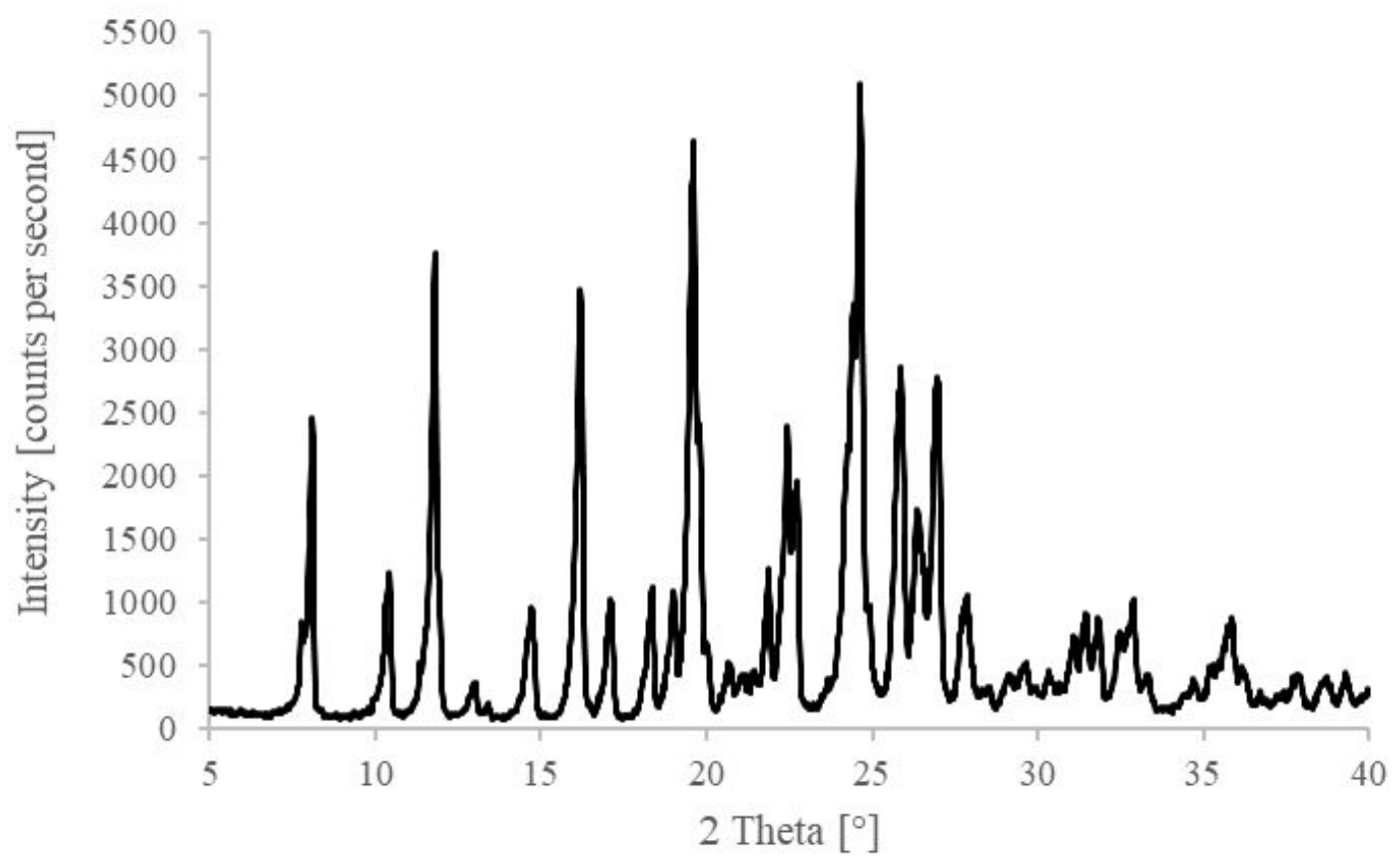

Figure S3. Characterization of the crystalline form of NIF by DSC and PXRD. (a) DSC heating curve of NIF as received. (b) PXRD pattern of NIF as received. 
(a)

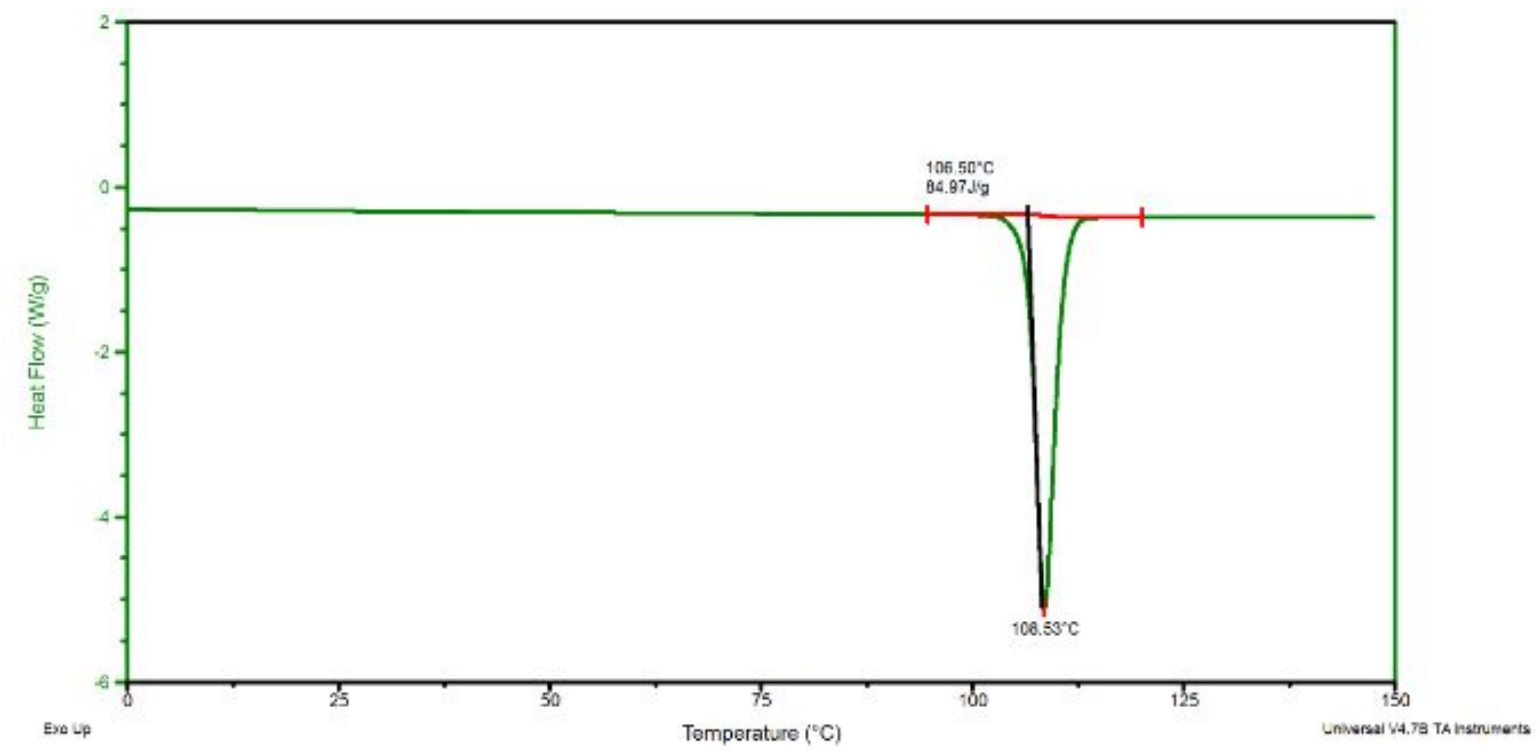

(b)

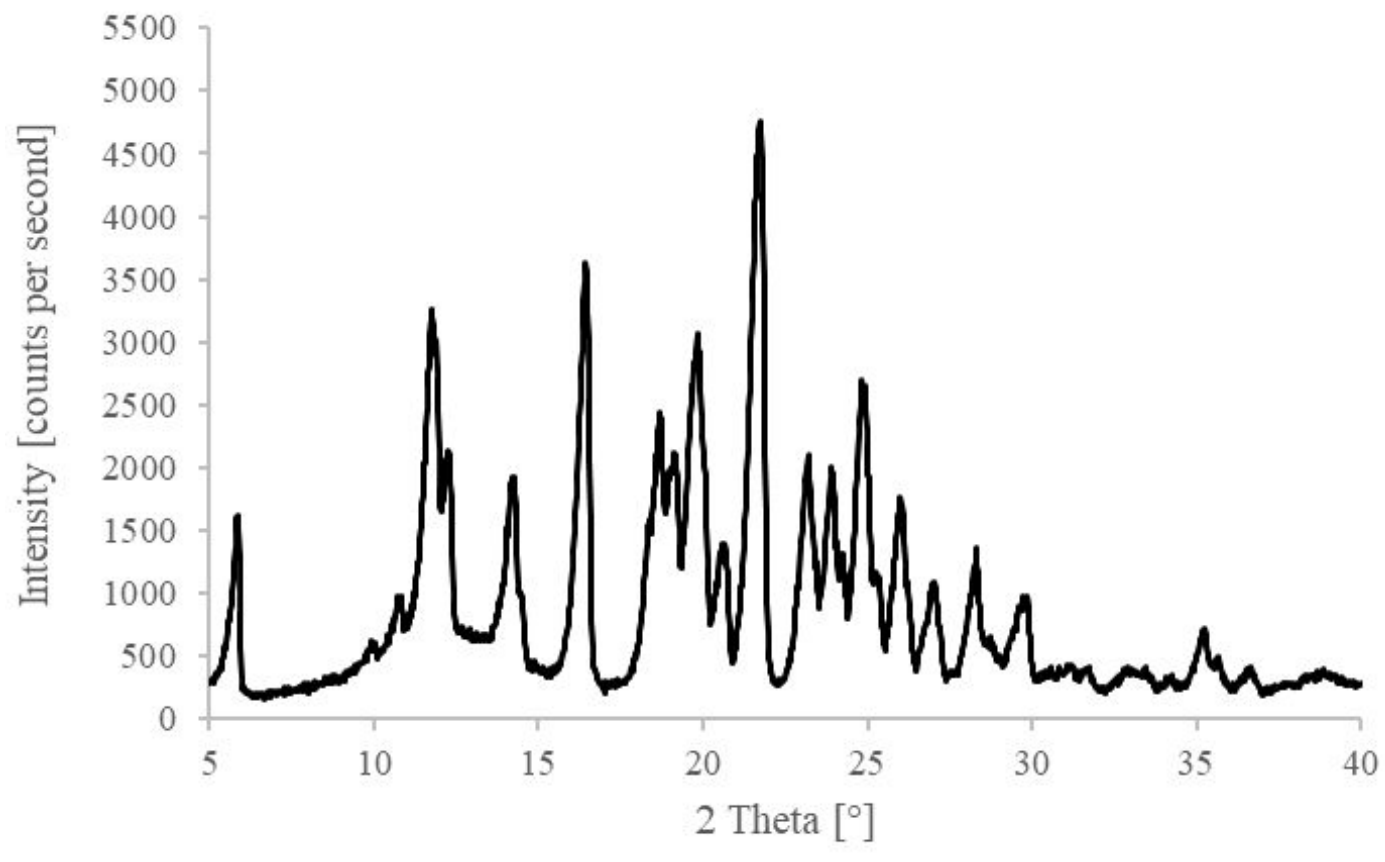

Figure S4. Characterization of the crystalline form of CLD by DSC and PXRD. (a) DSC heating curve of CLD as received. (b) PXRD pattern of CLD as received. 
(a)

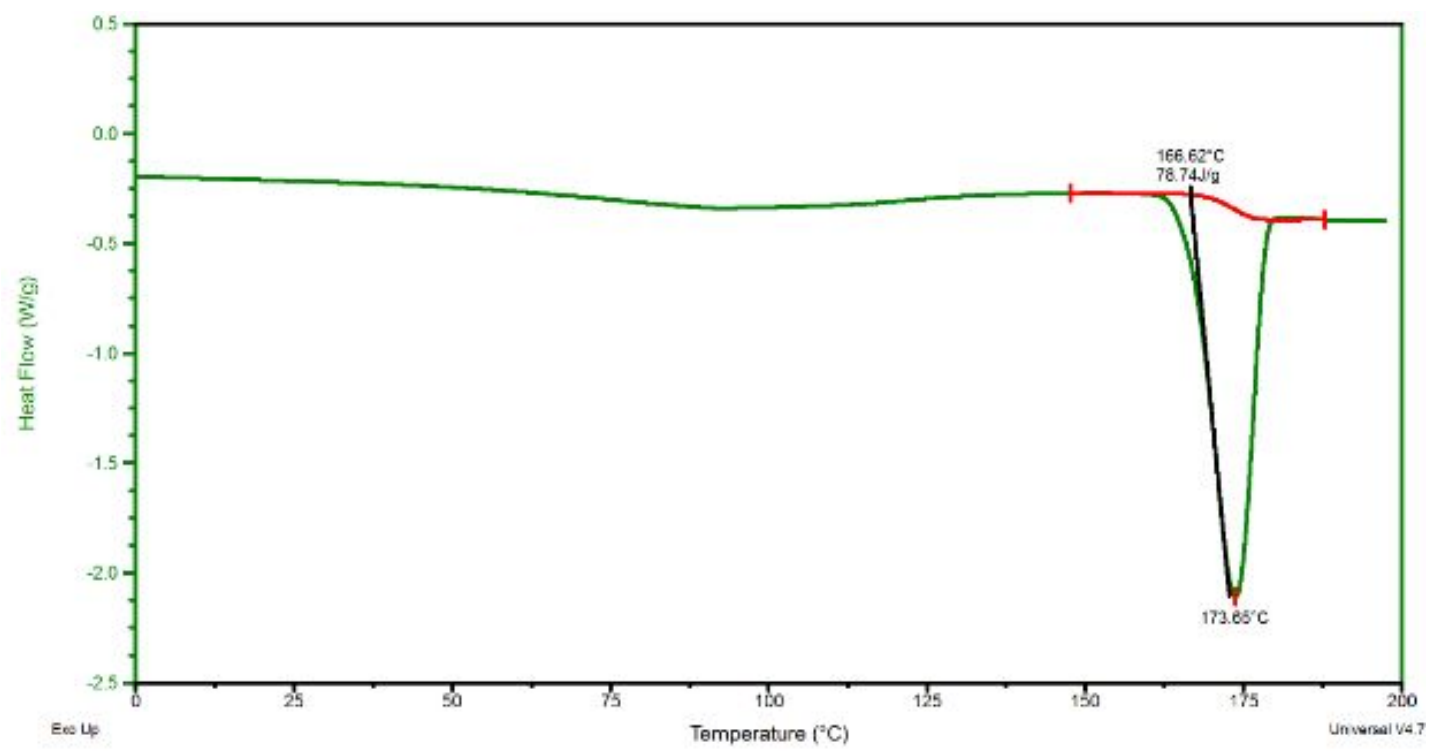

(b)

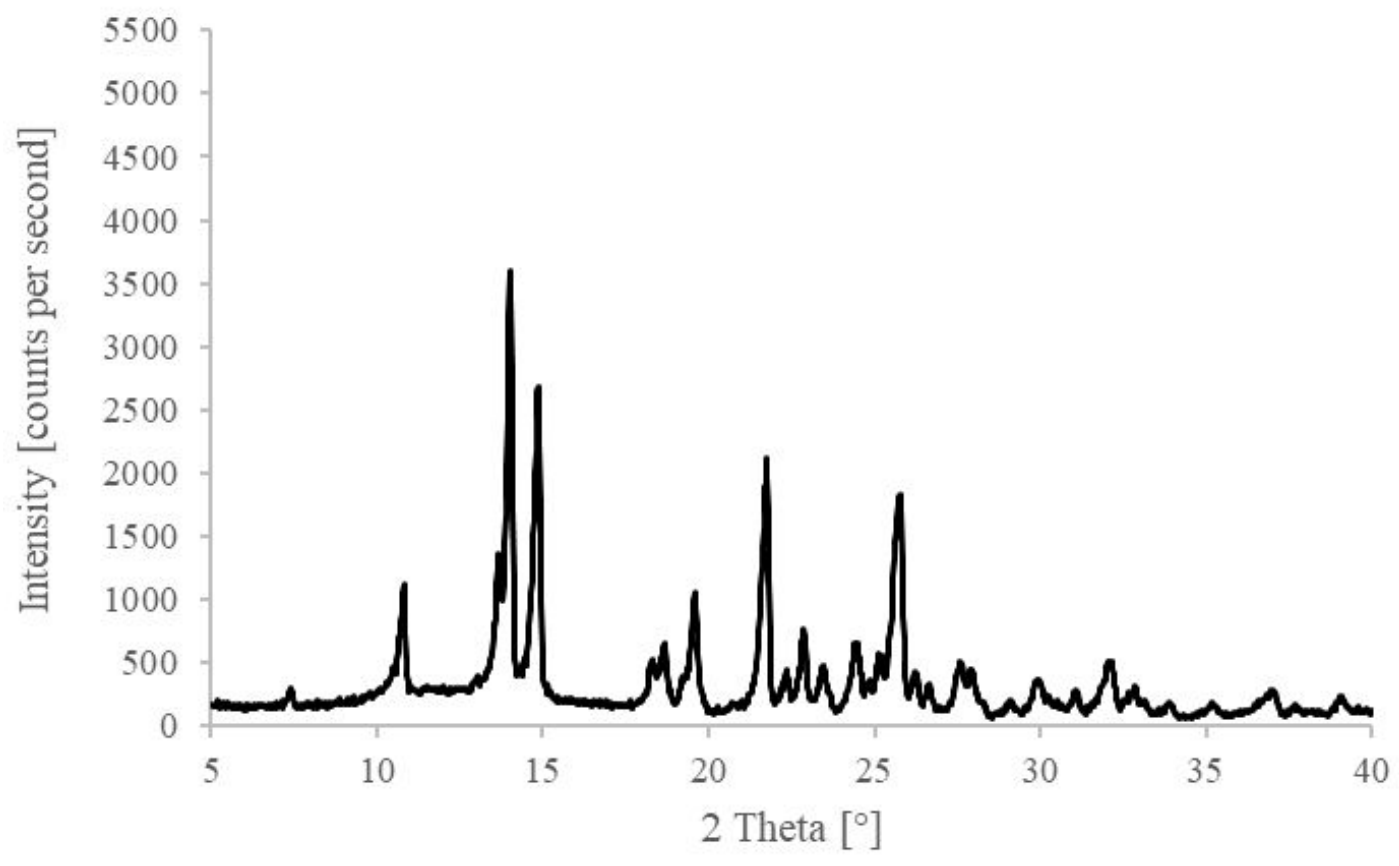

Figure S5. Characterization of the crystalline form of IPM by DSC and PXRD. (a) DSC heating curve of IPM as received. (b) PXRD pattern of IPM as received. 


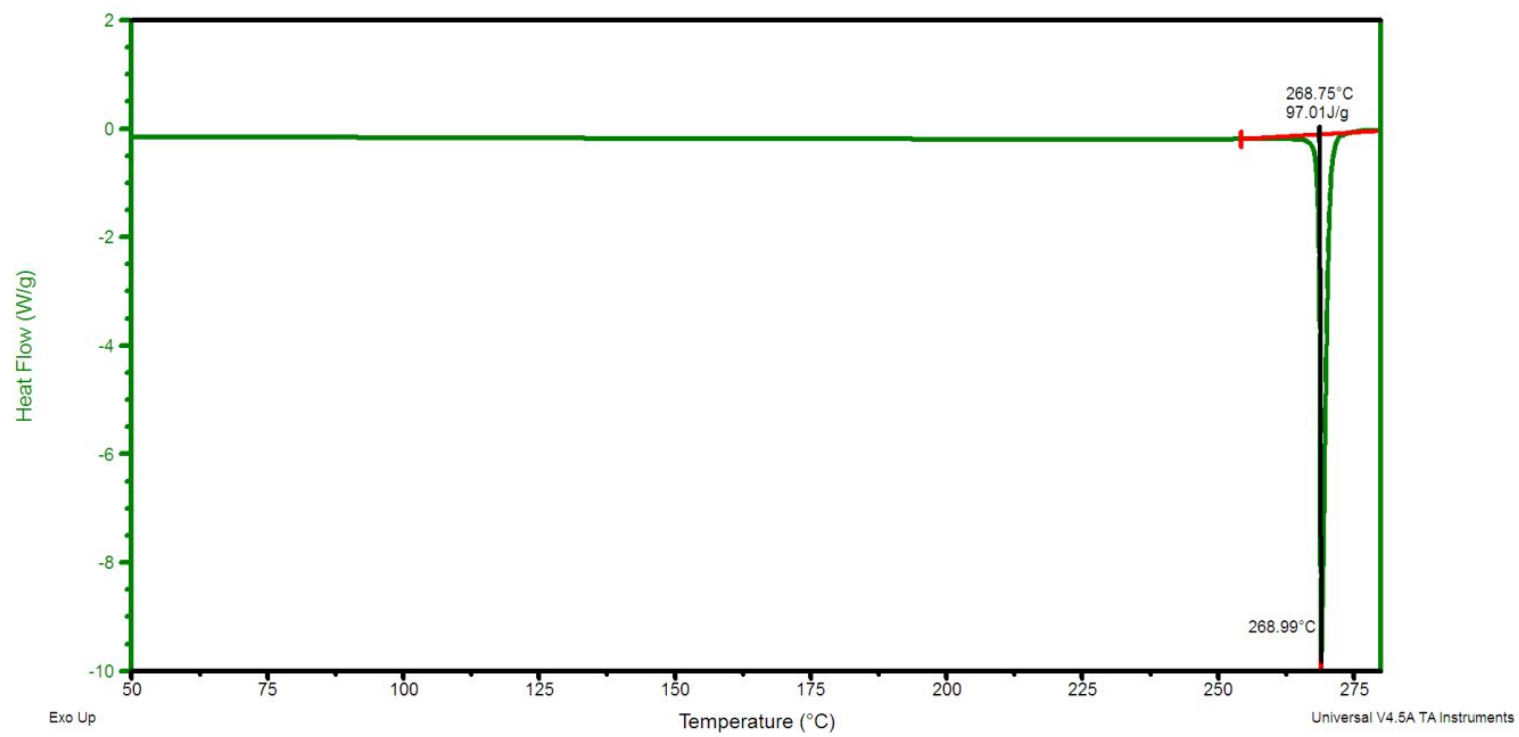

Figure S6. DSC heating curve of MTN as received. 


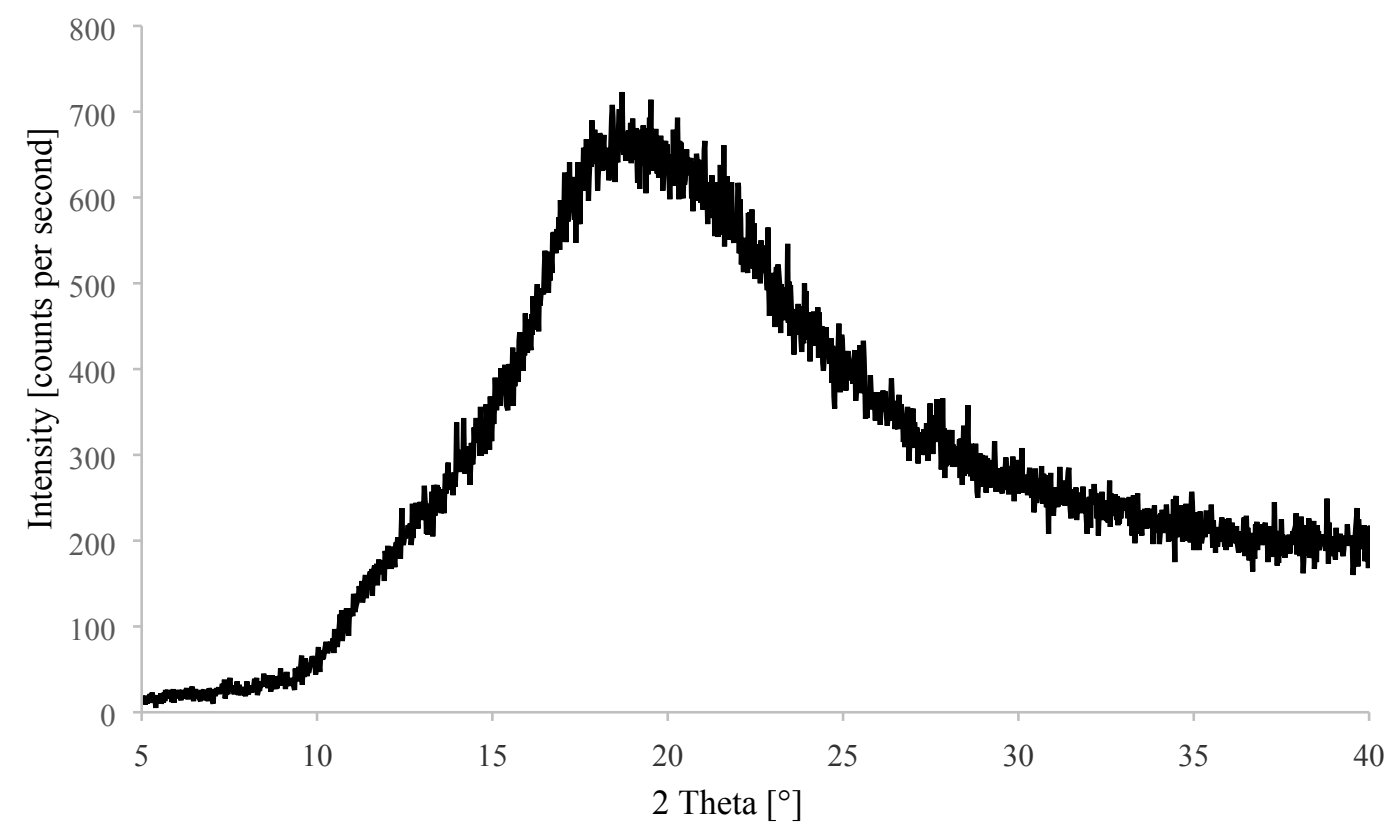

Figure S7. Characterization of the formulation containing 80\% HPMCAS and 20\% drugs (1:1 mole ratio of FDN and IPM) by PXRD. 


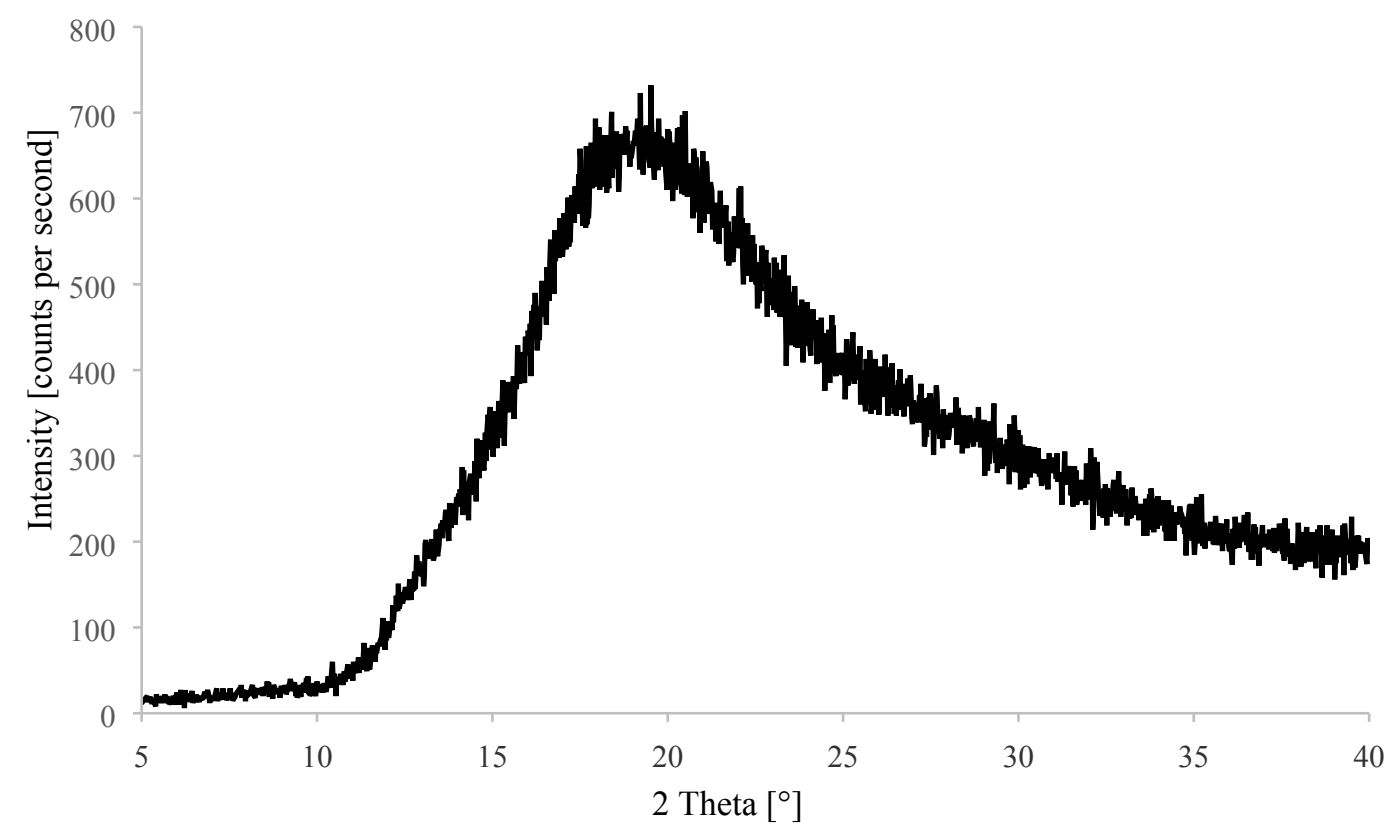

Figure S8. Characterization of the formulation containing $80 \%$ PVP and $20 \%$ drugs (1:1 mole ratio of FDN and IPM) by PXRD. 


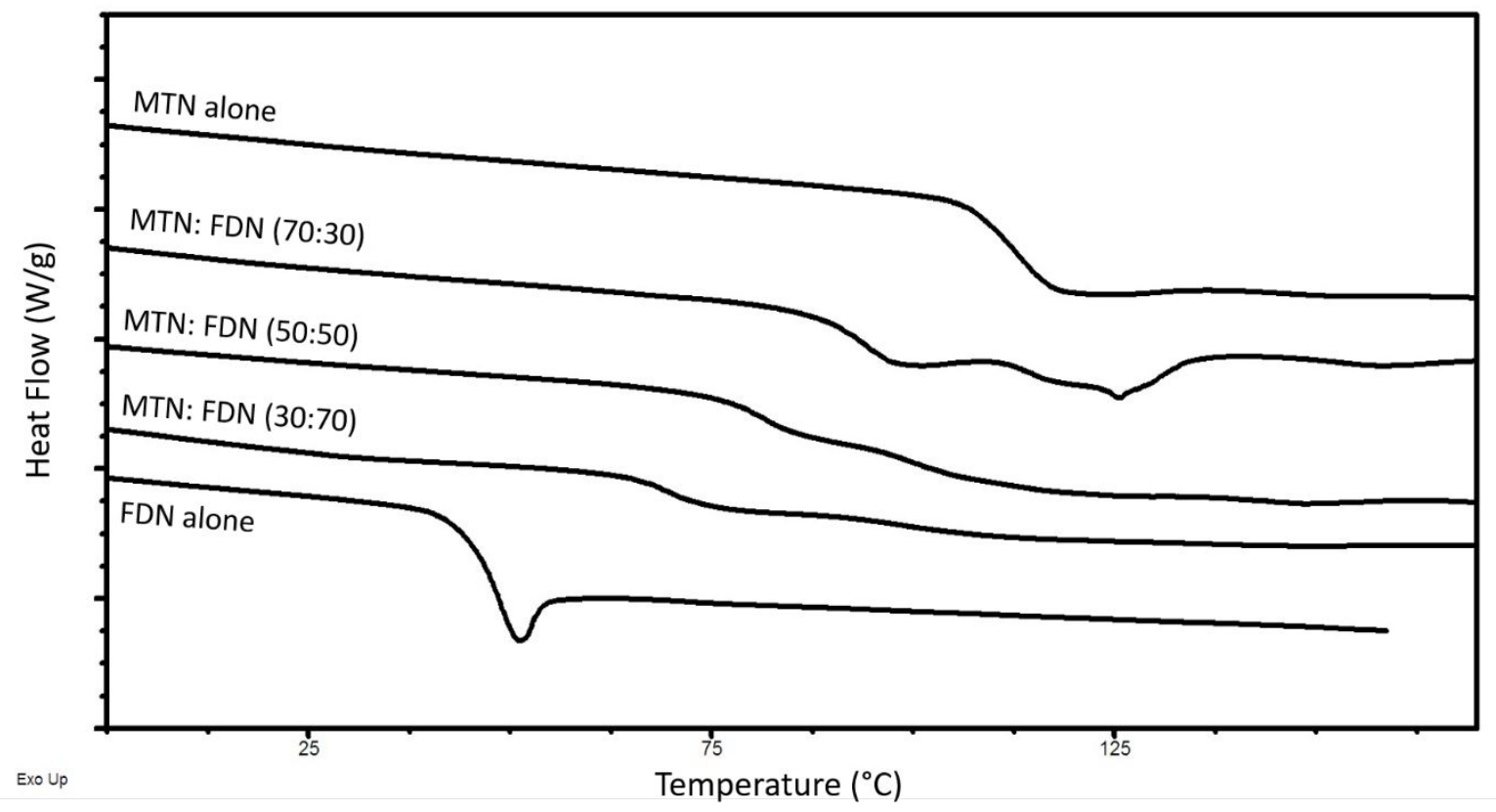

Figure S9. DSC thermograms of the FDN and MTN physical mixture at different molar ratios. 


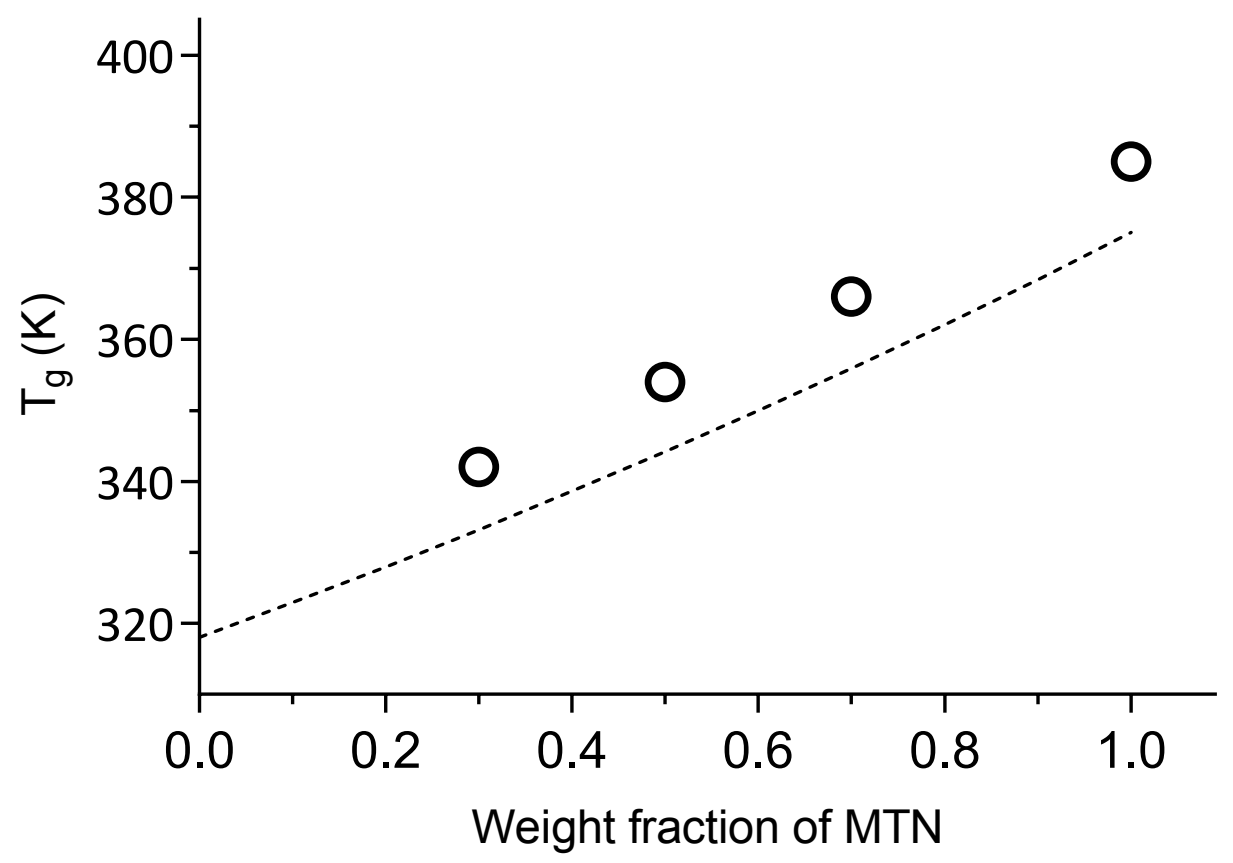

Figure S10. Glass transition temperature ( $\mathrm{T}_{\mathrm{g}}$ ) of a miscible system of FDN and MTN as a function of MTN weight fraction. Dashed line represents the predicted $\mathrm{T}_{\mathrm{g}}$ based on Fox equation. 


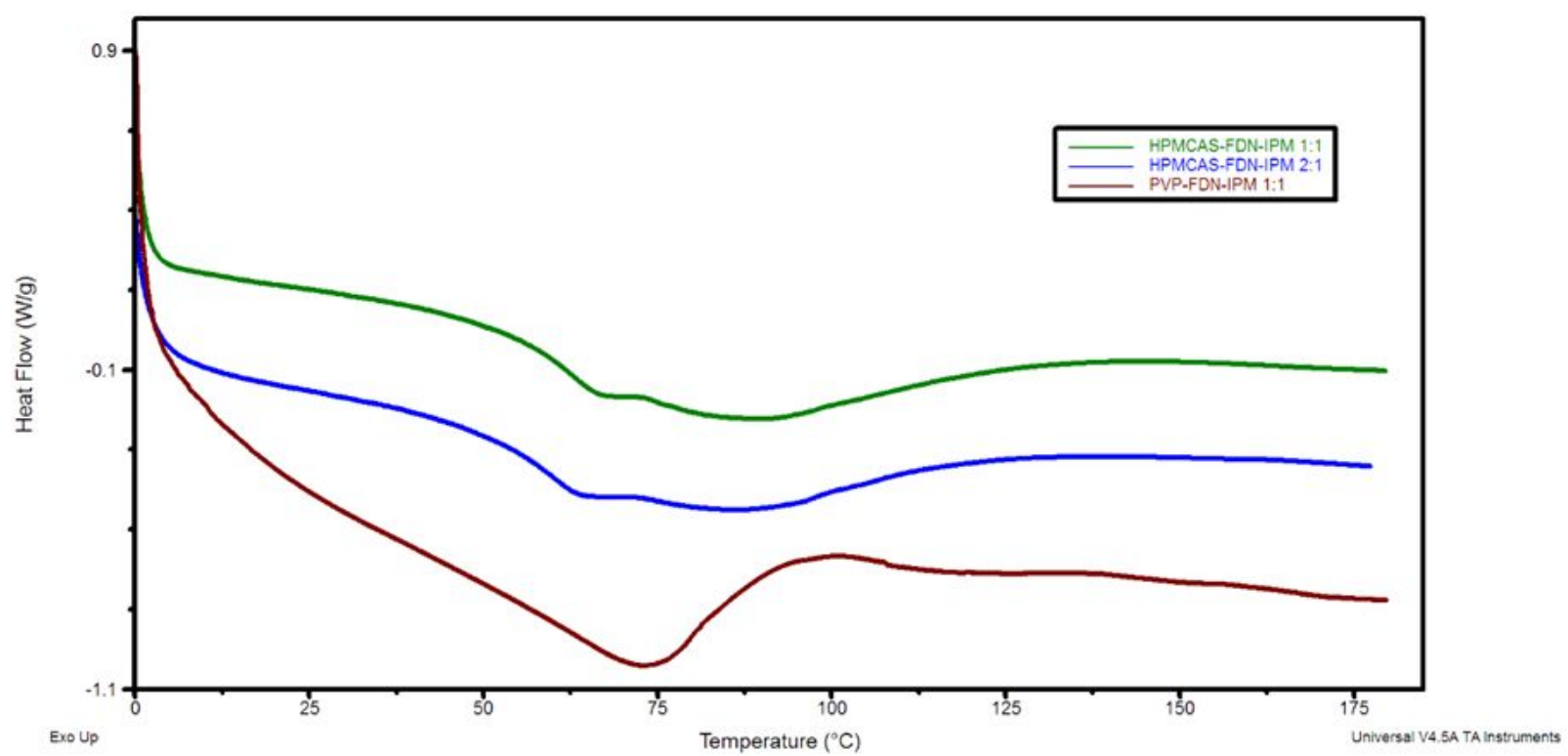

Figure S11. DSC heating curves of the HPMCAS-FDN-IPM 1:1 mole ratio (green curve), HPMCASFDN-IPM 2:1 mole ratio (blue curve) and PVP-FDN-IPM 1:1 mole ratio (maroon curve) at the end of the dissolution study. 
(a)

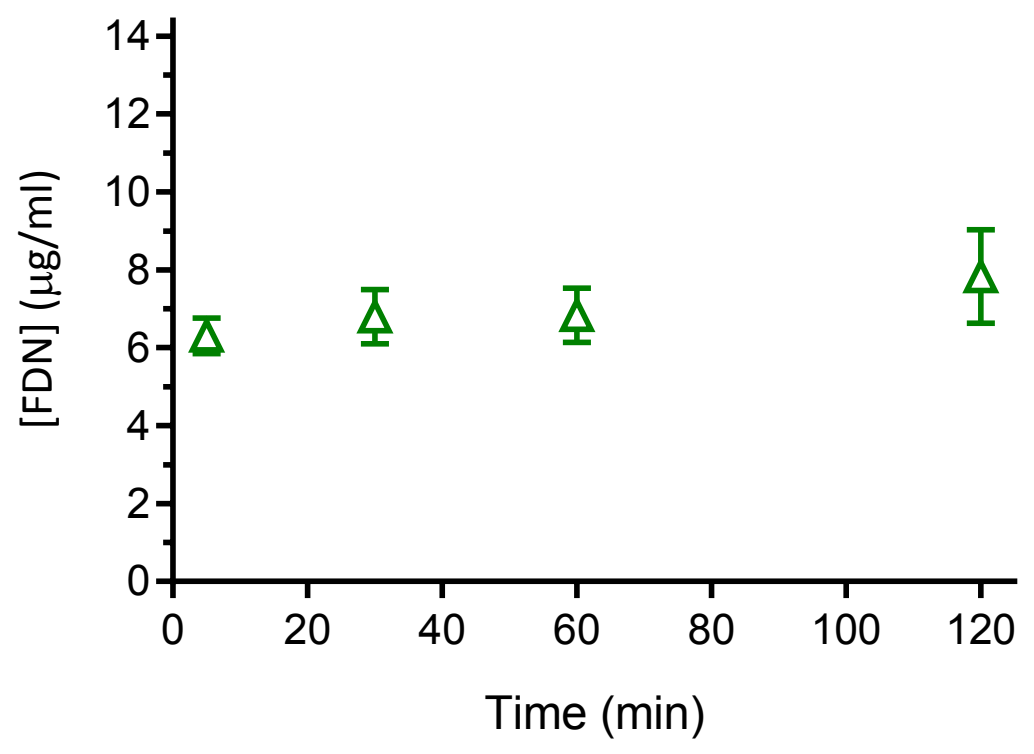

(b)

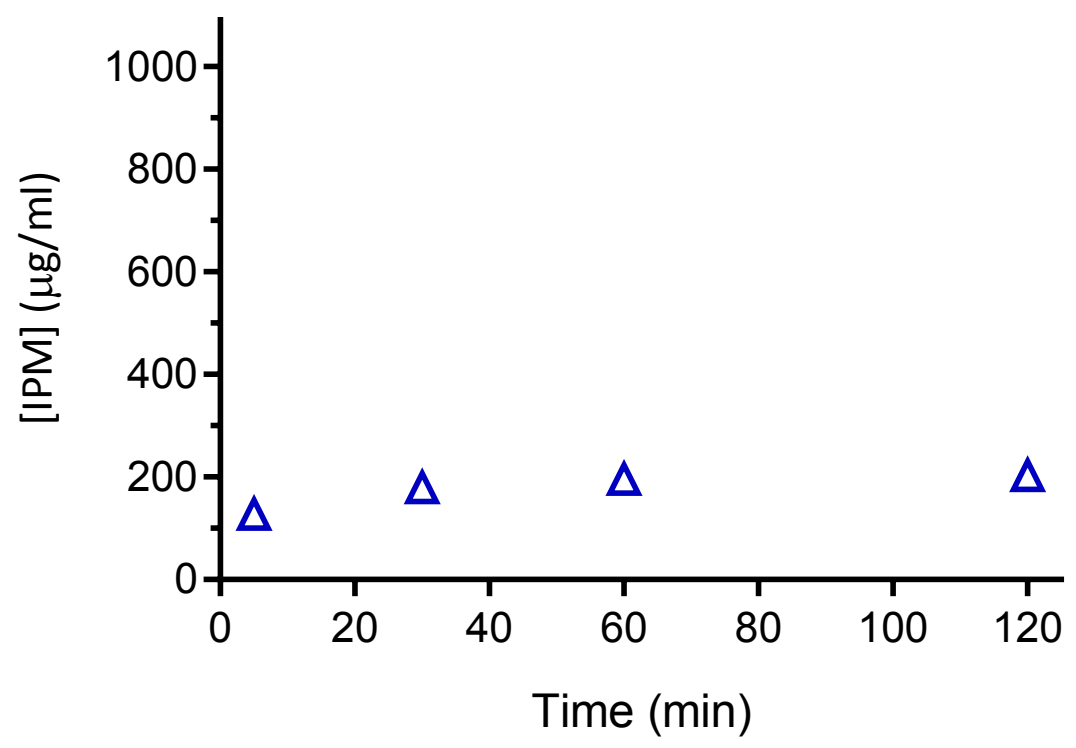

Figure S12. Dissolution profiles of formulations containing 80\% HPMCAS and 20\% drugs (2:1 mole ratio of FDN and IPM) in buffer (a) FDN and (b) IPM. Error bars show standard deviation, n=3. Error bars for some points are smaller than the symbols. 


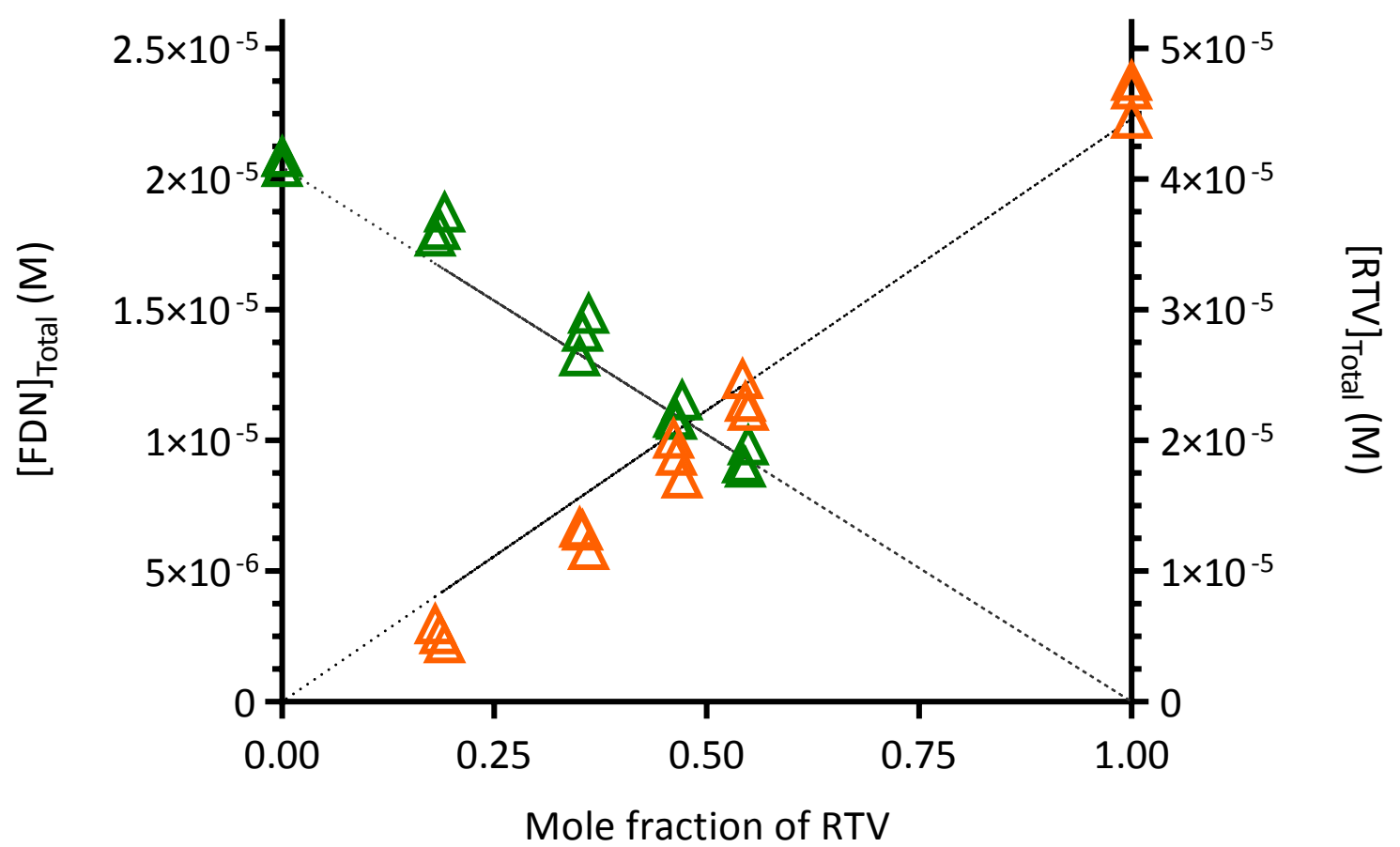

Figure S13. Concentration of FDN (green $\triangle$ ) and RTV (orange $\triangle$ ) in the supernatant layer using the antisolvent method as a function of the mole fraction of RTV in the solution. Dashed lines represent the predicted concentrations based on Eq. 2 (three replicates). 


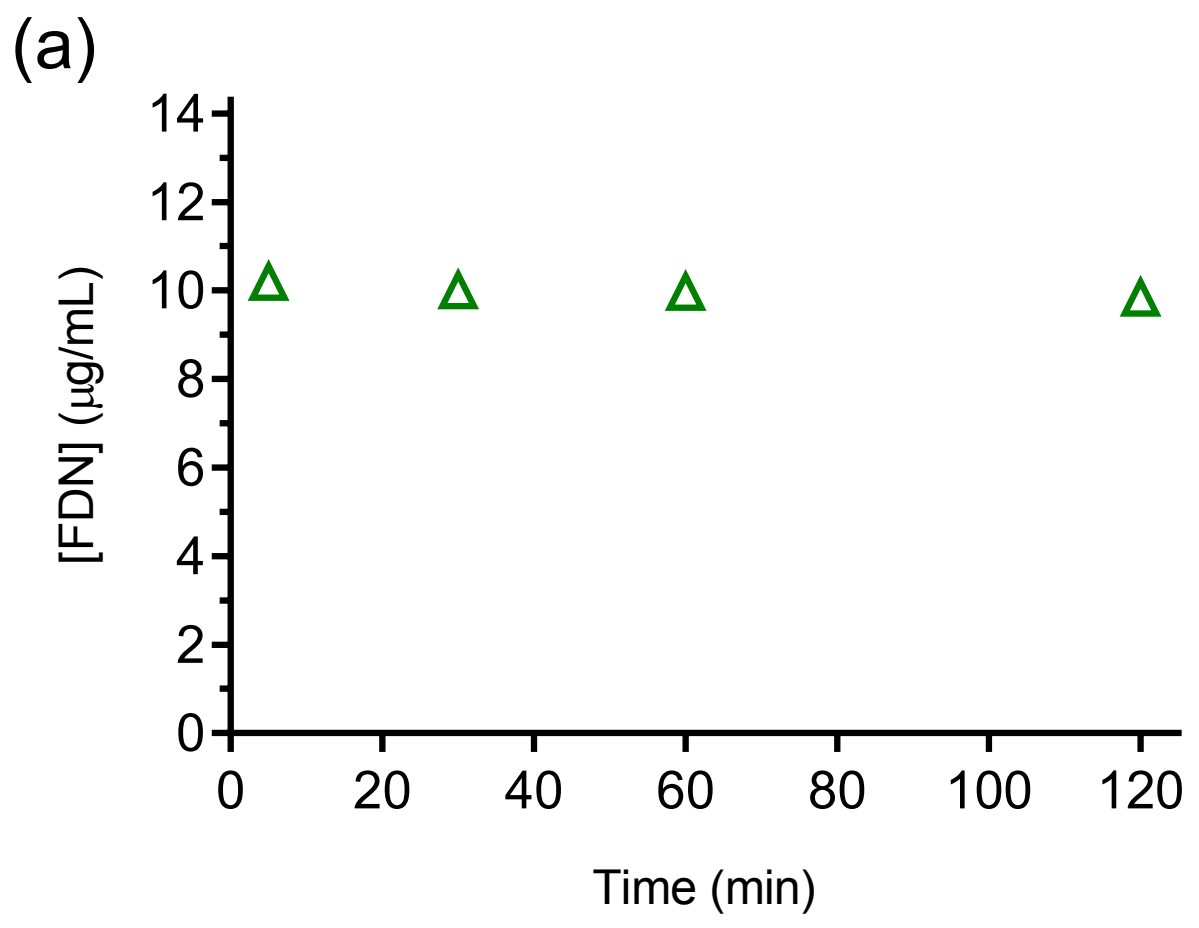

(b)

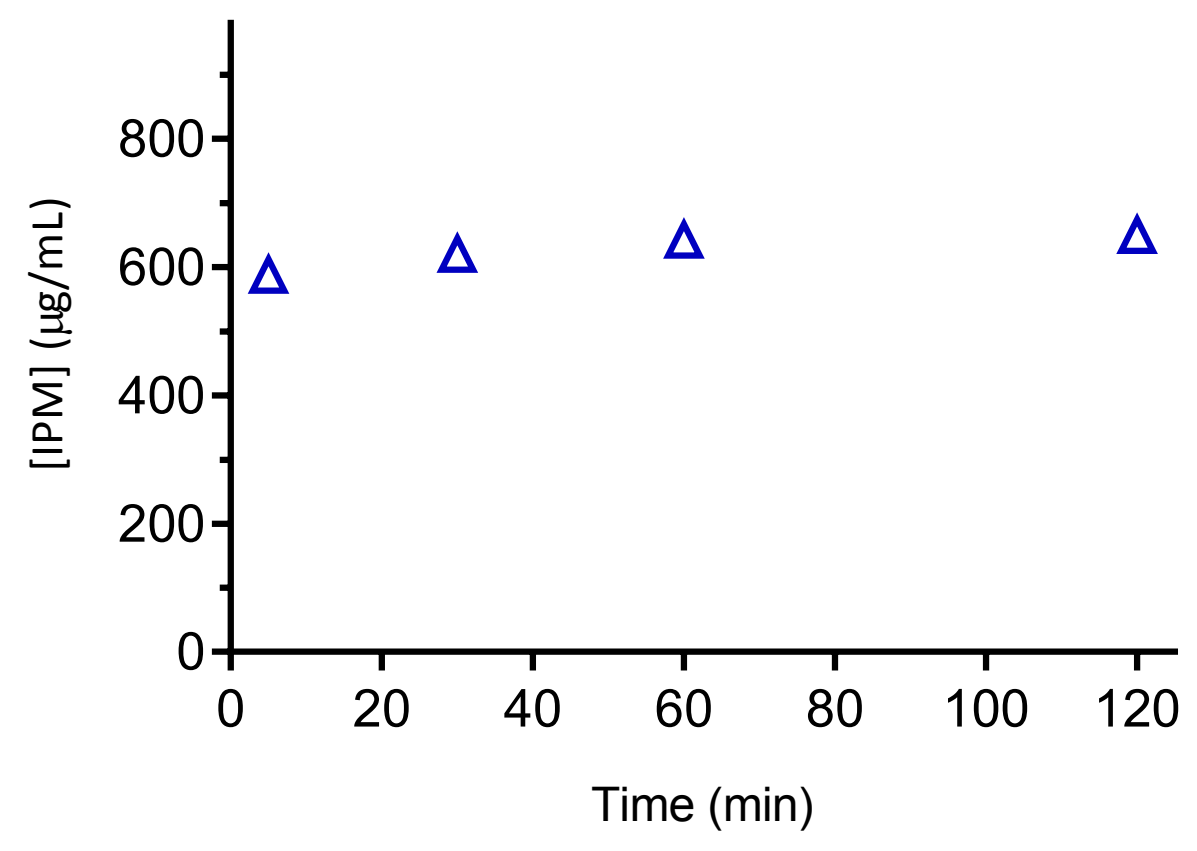

Figure S14. Dissolution profiles of formulations containing 80\% PVP and 20\% drugs (1:1 molar ratio of FDN and IPM) in buffer (a) FDN in green and (b) IPM in blue. Error bars (three replicates) show standard deviations (error bars are smaller than the symbols for some measurements). 